\title{
Ruta de aprendizaje de gestión del conocimiento en fundaciones empresariales
}

\section{Learning path of knowledge management in business foundations}

\author{
DOI: https://doi.org/10.17981/econcuc.40.2.2019.05
}

Artículo de investigación. Fecha de recepción: 17/02/2019 Fecha de aceptación: 24/07/2019

\author{
Amin Sinisterra-Núñez \\ Universidad Autónoma de Occidente-UAO. (Cali, Colombia) \\ amin.sinisterra@uao.edu.co \\ Lourdes Eugenia Osorio-Bayter \\ Universidad de La Salle. (Bogotá, Colombia) \\ losorio@lasalle.edu.co \\ Jesús Gabalán-Coello \\ Universidad Católica de Pereira-UCP. (Pereira, Colombia) \\ jesus.gabalan@ucp.edu.co \\ Fredy Eduardo Vásquez-Rizo \\ Universidad Autónoma de Occidente-UAO. (Cali, Colombia) \\ fvasquez@uao.edu.co
}

Para citar este artículo:

Sinisterra-Núñez, A., Osorio-Bayter, L., Gabalán-Coello. J. y Vásquez-Rizo, F. (2019). Ruta de aprendizaje de gestión del conocimiento en fundaciones empresariales. Económicas CUC, 40(2), 70-86. DOI: http://dx.doi.org/10.17981/econcuc.40.2.2019.05

\section{Resumen}

La gestión del conocimiento es hoy día un nuevo motor para el desarrollo empresarial, contar con estrategias claras que permita transmitir este conocimiento a generaciones de relevo resulta imprescindible, es por ello que este artículo presenta el diseño de una ruta de aprendizaje para la gestión del conocimiento en fundaciones empresariales con intervención en el departamento del Valle del Cauca (Colombia). Para lo cual se usó una metodología del tipo exploratorio-descriptivo. Se resalta como resultado el hecho de que existe un vinculo bastante claro entre las fundaciones estudiadas y las poblaciones a las que estas benefician. Se concluye que la gestión del conocimiento, para estas organizaciones, se encuentra estrechamente vinculada con la apropiación de experiencias, prácticas y lecciones aprendidas por cada colaborador.

Palabras clave: Gestión del conocimiento; fundación; conocimiento; información; aprendizaje

\begin{abstract}
Knowledge management is now a new engine for business development, having clear strategies that allow transmitting this knowledge to generations of success is essential, which is why this article presents the design of a learning route for management of knowledge in business foundations with intervention in the department of Valle del Cauca (Colombia). For which an exploratory-descriptive methodology was used. As a result, the fact that there is a fairly clear link between the foundations studied and the populations they benefit from is highlighted. It is concluded that knowledge management, for these organizations, is closely linked with the appropriation of experiences, practices and lessons learned by each collaborator.

Keywords: Knowledge management; foundation; knowledge; information; learning
\end{abstract}




\section{INTRODUCCIÓN}

La implementación de estrategias y procesos de gestión del conocimiento en las sociedades actuales, como motor de desarrollo, sustento de la nueva economía y base del sistema productivo, es una realidad palpable e innegable (Argyris, 1999; Vásquez y Gabalán, 2015). Parte de esta sociedad la constituyen las organizaciones, las cuales según Drucker (1996), deben tener un propósito y unos objetivos claros en función de dicho contexto, lo cual debe permitirles transitar y sobrevivir con éxito en un medio cada vez más competitivo, de lo contrario estarían condenadas al fracaso.

Es justamente en esta planificación organizacional que las entidades deben aprender a ser recursivas y a sobrellevar las carencias que se les presentan, teniendo que sortear problemas económicos, estructurales, sociales, políticos y de otra índole, los cuales les impiden aprovechar al máximo las posibilidades inherentes a esta nueva sociedad, llamada del conocimiento "enmarcada en la ética y el desarrollo humano en las organizaciones" (Quiñones y Senior, 2014, p. 13).

Sociedad, que exige a las organizaciones diseñar, desarrollar e implementar, dentro de su planificación y estructuración, procesos que les posibiliten capturar datos e información (Silva et al., 2019), de tal manera que puedan ser transformados en conocimiento, un conocimiento que combina los conocimientos tácitos, presentes en su Capital Humano conformante, con los conocimientos explícitos, siendo éstos aquellos tácitos que paulatinamente han podido trascender y aparecer como elementos propios, ya al servicio de la compañía.

Por tal razón, dichas organizaciones deben construir por sí mismas metodologías y desarrollar procesos que les permitan el flujo constante del elemento cognitivo, tan- to internamente, a través de las relaciones que en su conformación se establecen, como externamente, en términos de llevar su conocimiento a la sociedad y apropiar de esa misma sociedad conocimientos ausentes.

Todo ello mediante la educación, que en palabras de Hernández y Chumaceiro (2008):

[...] es el vehículo del conocimiento que se interpreta como la capacidad de entender los fenómenos humanos desde una perspectiva formal y científica según los criterios disciplinarios de cada profesión, facilita evaluar la realidad y posibilita dar las respuestas pertinentes, sobre todo cuando se enmarca en la interacción de los grupos sociales (p. 78).

Es en esta realidad organizacional donde surgen la creación y consolidación de fundaciones empresariales como estrategia de desarrollo asociada a potenciar el aspecto externo señalado, debido a que son éstas las que se pueden encargar de realizar las intervenciones sociales por parte de la empresa, y de transferir y apropiar el know-how respectivo, de manera bidireccional, para que sea aprovechado, tanto por ellas como por su contexto circundante. $\mathrm{Al}$ respecto, la Fundación Carvajal (2016) establece como el fortalecimiento de las fundaciones empresariales, aunado a procedimientos de gestión del conocimiento, posibilita el mejoramiento continuo, el desarrollo de las competencias, la calidad y la replicabilidad de las intervenciones exitosas, constituyéndose en sí mismo un propósito estratégico.

De esta manera, el presente artículo sugiere entonces una metodología que contribuya a que las fundaciones empresariales del Valle del Cauca, Colombia, definan una ruta de aprendizaje, basada en la gestión del conocimiento, y potencie la calidad de sus intervenciones sociales, y al mismo tiempo, le permita a las fundaciones apropiar el conocimiento social en función del propósito y 
los objetivos estratégicos de la empresa de la cual forman parte.

Esto último constituye un aspecto importante al considerar a las empresas como organismos vivos y sinérgicos, donde sus fundaciones deben considerarse como parte activa de dicho conjunto. Por lo tanto, en materia de gestión del conocimiento, deben diseñarse, definirse y registrarse procesos de índole sistémico que permitan a dichos componentes adherirse, de manera aportante, a la estructura organizacional.

\section{FUNDAMENTACIÓN TEÓRICA}

En las últimas décadas se han acelerado drásticamente las condiciones necesarias para producir cambios tanto en los negocios como en la sociedad, al respecto Villasmil y Crissien (2015) comentan como esta última década se "ha caracterizado por cambios vertiginosos desde perspectivas políticas, económicas, sociales y de mercado, que obligan a las organizaciones a emprender nuevas maneras de hacer las labores" (p. 246), $\mathrm{y}$ "estos cambios han derivado nuevas formas de producción, intercambio y conexión globalizada; así como una reorganización y concepción de las prácticas" (Chumaceiro, Hernández, Meléndez y Acurero, 2017, p. 201-202)

Una de las consecuencias de estos cambios radica en como los procesos sociales que las empresas realizan son ahora más importantes en la consecución del éxito organizacional. Sin embargo, este campo empresarial no ha sido poco explorado, dado el afán mismo del negocio, el cual exige que se prioricen mayormente los aspectos relacionados con el mercado, situación que dista del concepto de fundación (Villar, Butcher y Gandini, 2014), donde el aspecto social se convierte en su esencia, y en muchos casos, es ajeno en sí mismo a la generación de riqueza.
Es aquí donde la gestión del conocimiento aparece como proceso apalancador para mejorar el desempeño de las intervenciones sociales empresariales, a partir de la explicitación de sus experiencias y lecciones aprendidas, en procura de sacar un mejor provecho de la organización, incluso monetario, de los conocimientos tácitos presentes en sus capitales conformantes, especialmente humano y relacional (Awad, 2007).

En ese sentido, Gorelick, Milton \& April (2004) afirman que el aprendizaje y la gestión del conocimiento no son fines en sí mismos para las fundaciones empresariales, sino que son medios para mejorar la efectividad de sus prácticas y asegurar para cada decisión tomada en un proceso de intervención social se realice teniendo en cuenta el conocimiento y la experiencia existente, en beneficio de la sociedad y la empresa.

Aquí, es importante señalar, aunque gestión del conocimiento es un término de uso generalizado (Piorun, 2018), existen evidencias de como el significado y los enfoques que del mismo se tienen en el contexto de las organizaciones es muy diverso, lo cual dificulta su real comprensión y la precisión de criterios, tanto al momento de implementar programas en este campo como en el de evaluar su impacto. Y esto aplica también para su interpretación en el contexto de las fundaciones.

Sin embargo, a través de diversos autores (Toffler, 1990; Barker, Thorne \& Dutnell, 1997; McAdam \& Reid, 2001; Soret, 2007; Castells, 2009; Briceño y Bernal, 2010) se intenta generar una definición conceptual en dicho contexto, lo cual permite precisar como el conocimiento es para estas entidades un recurso estratégico asociado a una nueva sociedad, necesitada de su efectiva gestión para poder plasmar y aprovechar la multiplicidad de relaciones establecidas entre la empresa y los sujetos internos y ex- 
ternos, en procura de una real transformación, tanto de los individuos como de la empresa y de su ámbito social de desempeño.

De esta manera, se puede hablar de algunos enfoques de la gestión del conocimiento que pueden soportar dicha definición, siendo éstos: a) sociocultural (Barragán, 2009), el cual impulsa una cultura organizacional que promueve cambios en la actitud, confianza, creatividad, colaboración, comunicación y conciencia del valor del conocimiento entre los miembros de una comunidad; b) filosófico (Kakabadse, Kakadse y Kouzmin, 2003), que aborda la epistemología del conocimiento para explicar la realidad social; c) en red (Londoño y Castillo, 2013), el cual tiene que ver con el pensamiento sistémico y estudia las relaciones y flujos de conocimiento presentes en los conjuntos sociales, cualquiera sea su nombre o características.

Adicional a éstos siguen: d) comunidades de práctica (Wenger, McDermott \& Snyder 2002; Olmos, Castro \& D'este, 2014), basado en cómo el conocimiento intrínsecamente constituye una propiedad común entre una sociedad, para construir aprendizajes colectivos (implica compromiso mutuo y participación colectiva); e) social (Canals, 2002), que enfatiza en la importancia de los aspectos sociales en el momento de promover la creación o la compartición del conocimiento, y f) historias de aprendizaje (Marín y Velasco, 2001), que tiene relación con la generación de procesos de reflexión y aprendizaje a partir de las experiencias de la gente.

Todos estos enfoques ponen de manifiesto las relaciones que se establecen y deben establecerse en un sistema como el expuesto en la presente propuesta, donde la labor de la fundación consisten en establecer nexos cognoscitivos entre la empresa y su comunidad o sociedad de influencia (Carcel, 2014).
En esta misma dirección, Choo (1999) afirma como las relaciones de la organización con sus externos deben sostenerse a través del tiempo y generar la mayor cantidad de conocimiento posible, por lo cual deben ser flexibles, dinámicas y contar con estructuras sociales que generen espacios para compartir dichos conocimientos, a partir de la interacción, el intercambio y la creación de elementos cognoscitivos conjuntos.

Es así, como, en el caso concreto de Colombia, existen algunos ejemplos de empresas que promueven la gestión del conocimiento a través de sus propias fundaciones, las cuales más que pretender hacer parte de la sociedad del conocimiento por simple inercia, asumen este proceso como una manera real de afianzar las relaciones establecidas con la sociedad e incorporar en su memoria organizacional conocimientos comunes (Quadrado y Simone, 2011), carentes de procesos efectivos de explicitación por su misma condición históricamente han.

Tal es el caso de la Fundación Promigas (2015), donde se considera la gestión del conocimiento como una estrategia transversal en todas sus iniciativas de cambio organizacional y social (Sukier et al., 2018), o de la Fundación Saldarriaga Concha (2017), donde se enfoca la gestión del conocimiento en el registro ordenado de buenas prácticas comunitarias y sociales y de lecciones aprendidas.

Ya para el ámbito regional, en el departamento del Valle del Cauca, se encuentra la Fundación Carvajal (2016), la cual fortalece sus procesos de gestión del conocimiento a través del mejoramiento continuo y el desarrollo de competencias de sus colaboradores, por medio de comunidades de práctica (Wenger, 2001; Lesser \& Storck, 2001) y explicitación y transferencia de historias de aprendizaje y buenas prácticas (Valencia, 2013), actividades que muestran el éxito de sus intervenciones sociales. 
Como se puede apreciar, existe un interés creciente por analizar los procesos de gestión del conocimiento en las fundaciones empresariales, lo que constituye una tendencia global; sin embargo, en Colombia, estas actividades están apenas dando sus primeros pasos hacia su consolidación (Asociación de Fundaciones Empresariales y FamiliaresAFE, 2012), pensando en la gestión del conocimiento como herramienta aportante al desarrollo organizacional y a la inclusión social de la labor de la empresa.

\section{MetodoloGía}

El alcance del estudio es de tipo exploratoriodescriptivo. Exploratorio examinar un tema poco estudiado y descriptivo por caracterizar dicho fenómeno (Hernández, Fernández y Baptista, 2014), y a partir de estos hechos plantea una ruta de aprendizaje, como propuesta metodológica basada en la gestión del conocimiento, para el posicionamiento y progreso organizacional y social de las fundaciones empresariales (King, 2009) y Stary (2016), para quienes las rutas de aprendizaje permiten la difusión eficaz de la información y su transición hacia el conocimiento, en términos de detectar y promover las mejores prácticas en entornos determinados).

Al mismo tiempo, emplea el método deductivo para plantear un conjunto de conceptos, principios, definiciones y enfoques asociados al ámbito empresarial y al de la gestión del conocimiento, para luego plantear unas conclusiones específicas que permitan verificar la validez de la metodología propuesta.

Dicha metodología parte de un diseño de enfoque cualitativo, soportado en el tratamiento de datos procedentes de fuentes de información diversas (principalmente primarias y secundarias). Es decir, una metodología que se soporta en el contacto directo de los investigadores con las fundaciones em- presariales y con las comunidades, llevando a cabo diálogos participativos a través de los cuales se construyen rutas de aprendizaje desde las que se adquiere, transmite y apropia información con la finalidad de ser transformada en conocimiento, a partir de su explicitación (Davenport \& Prusak, 2001; Portillo, 2015), apalancándose en sus propias dinámicas relacionales y operacionales.

De esta manera, se presenta el tamaño de muestra constituido por 11 fundaciones empresariales, 10 con sede en el Valle del Cauca y una (1) ubicada en Bogotá, pero con alta injerencia en el departamento objeto de estudio. Para la selección de esta muestra se tuvieron en cuenta características relacionadas con el tamaño, principalmente, pero también con trayectoria (historia) y posibilidad de acceso a su información.

Es así como se emplean diferentes técnicas para la recolección de dicha información, tales como la revisión documental organizacional y comunal, la entrevista y la encuesta (estas últimas aplicadas a las empresas, fundaciones, comunidades y expertos); mecanismos utilizados para identificar y reconocer las prácticas realizadas por las fundaciones empresariales a analizar.

En el caso de las encuestas y entrevistas, se componen de preguntas abiertas y cerradas, cuyo objetivo es identificar ¿qué conocen las fundaciones y sus colaboradores en relación con la gestión del conocimiento?, ¿cuáles elementos asociados a esta práctica consideran fundamentales?, ¿de qué manera implementan procesos de gestión del conocimiento?, y ¿qué herramientas gerenciales o administrativas conocen y/o emplean para esta labor?, entre otras.

De esta forma, el estudio se soporta completamente en el trabajo de campo realiza- 
do, lo que reafirma su carácter cualitativo, donde la metodología descrita intenta reconocer in situ, por medio de la participación activa de los sujetos, entidades y comunidades objeto de análisis, el quehacer rutinario que realizan, con la intención de ubicar en dichas prácticas elementos de la gestión del conocimiento y sugerir posibles rutas de acción en dirección a su gestión, vista como una opción para el desarrollo, basado en el componente cognitivo, de las organizaciones y de los factores asociados a su contexto (Mori, 2008).

Por tanto, la mayor relevancia de este trabajo radica más en los resultados presentados a continuación que en los mismos métodos y materiales empleados, pues el trabajo cualitativo realizado no se aleja mucho de los mecanismos para tal fin reconocidos y habitualmente implementados, mientras que el valor diferencial del presente artículo sí estriba en lo que se encuentra luego del procedimiento señalado.

\section{Resultados}

En primera instancia, se debe decir como las intervenciones sociales realizadas por las fundaciones empresariales estudiadas tienen una estrecha relación con la población que intentan sea beneficiada. Una revisión inicial de los documentos empresariales y comunales permite identificar en este contexto algunos elementos asociados a la gestión del conocimiento (unos más evidentes que otros), siendo principalmente:

- Capital humano. Entendido en este ámbito como aquellas personas que fungen como colaboradores y empleados de las fundaciones y que poseen aquellos conocimientos tácitos que necesitan ser explicitados en la relación fundación empresarial - comunidad (Hausmann, 2013).
- Flujo de información. Es el ir y venir del insumo más importante de la gestión del conocimiento: la información, donde dicha identificación y su posterior gestión posibilitan ubicar las relaciones informacionales existentes (y con posibilidad de existencia) entre la fundación empresarial y la comunidad, reconociendo las actuales y futuras asociaciones sistémicas (Schoemaker y Tetlock, 2017).

- Medios de interacción. Activos tangibles e intangibles de interacción (Goh, 2002), utilizados por el capital humano para el tratamiento y flujo de la información (archivos, documentos, hardware, software, redes de comunicación, etc.).

- Conocimiento tácito. Aquel conocimiento que se encuentra en la mente de las personas y que necesita ser explicitado, a través de diferentes prácticas y medios, para poder asumirse como conocimiento organizacional (Máynez y Cavazos, 2011).

Con base en estos conceptos, se logró comprender y, posteriormente, verificar las formas como las fundaciones empresariales realizan sus procesos asociados a la gestión del conocimiento, las cuales deben ser distintas a las maneras en que las organizaciones tradicionales realizan dicho proceso, dadas las características concretas ya expuestas.

De esta forma, se tiene que para las fundaciones analizadas, el primer tema abordado consistió en reconocer qué tanto saben de la gestión del conocimiento.

Como se puede apreciar en la Figura 1, la mayoría de las fundaciones (90,9\%) afirma saber qué es la gestión de conocimiento. La única fundación que no contestó afirmativamente expuso como razón: la confusión conceptual entre dato, información y conocimiento, pero reconoce que existe el elemento cognitivo, así no desarrolle aún procedimientos para su gestión. 


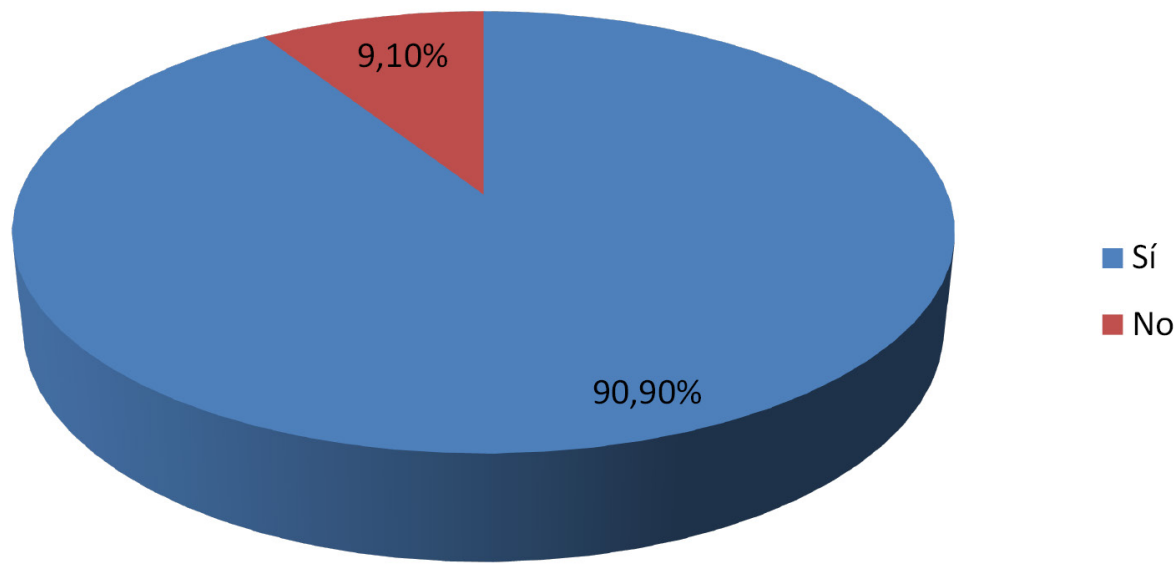

Figura 1. ¿Qué tanto saben las fundaciones empresariales sobre la gestión del conocimiento? Fuente: Elaboración propia.
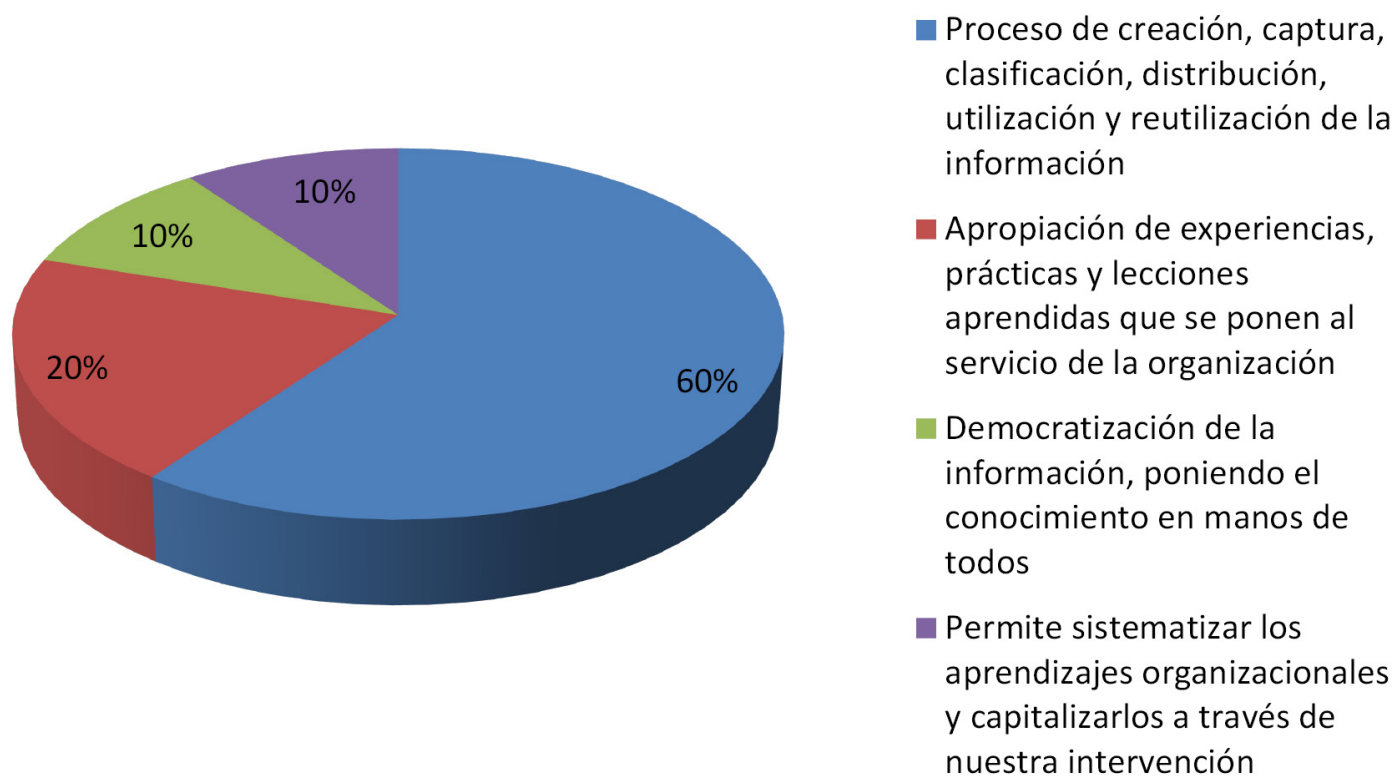

- Permite sistematizar los aprendizajes organizacionales y capitalizarlos a través de nuestra intervención

Figura 2. Definiciones específicas acerca de la gestión del conocimiento.

Fuente: Elaboración propia.

En este orden de ideas, cuando se les pide definir concretamente qué es dicho concepto, el $60 \%$ de las que contestaron afirmativamente menciona que es un proceso asociado a la información que trasciende hacia un nuevo elemento, el cual involucra su creación, captura, clasificación, distribución, utilización y reutilización, posición coincidente en gran parte con Nonaka y Takeuchi (1999). Las restantes fundaciones entregan diversas definiciones, las cuales pueden revisarse en la Figura 2.
La Figura 3 muestra los elementos, que según las fundaciones son trascendentales para una eficaz gestión del conocimiento (llama la atención el poco porcentaje asignado a la retención de los colaboradores, que se supone debería ser un aspecto importante para las fundaciones). Dentro del $15,4 \%$ que se clasifica como "otros" se mencionan: preservación del capital intelectual, motivación, equipos interdisciplinarios y valoración del conocimiento como activo de valor. 

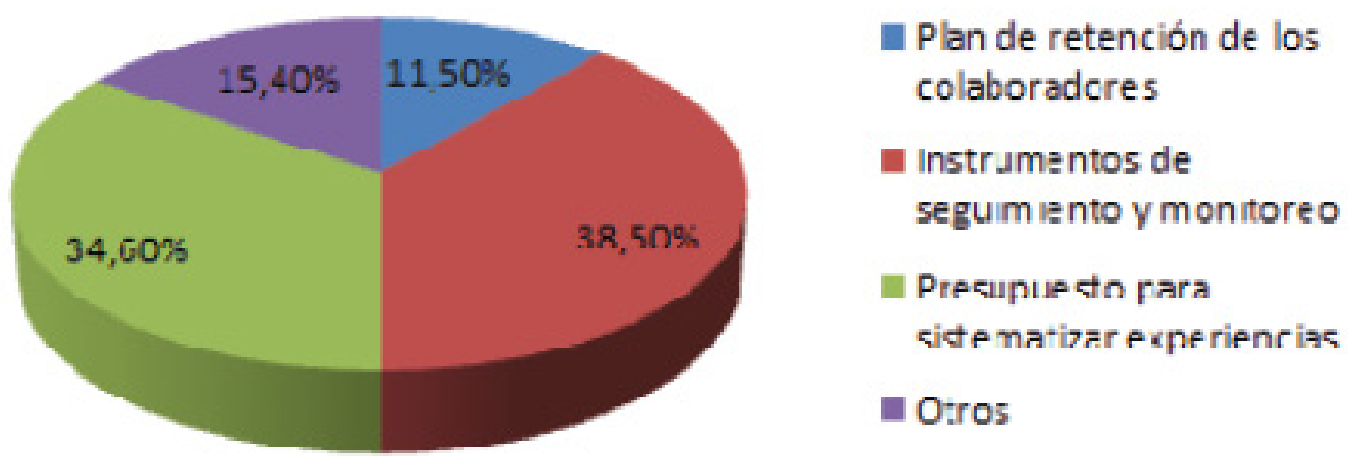

Figura 3. Elementos fundamentales para la gestión del conocimiento.

Fuente: Elaboración propia.

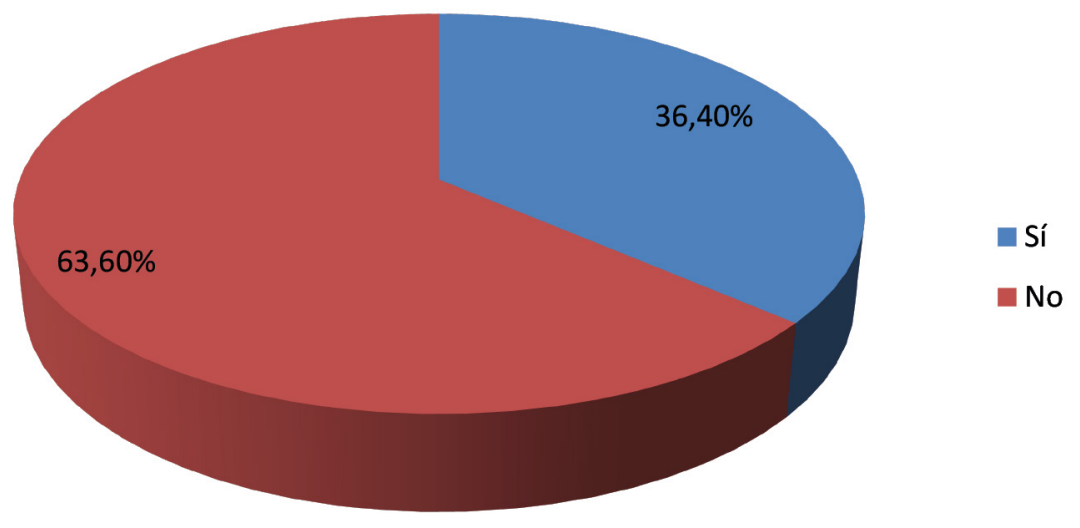

Figura 4. La gestión del conocimiento como proceso gerencial o administrativo.

Fuente: Elaboración propia.

En la Figura 4 se expone la opinión de las fundaciones, en términos de si consideran o no a la gestión del conocimiento como un proceso fundamental para el ente gerencial o administrativo.

Aquí, llama la atención como tan solo el $36,4 \%$ dice que sí, especificando como procedimientos de gestión del conocimiento los siguientes: documentación y sistematización de procesos, generación de resultados y productos intelectuales con base en dicha gestión, incorporación de la gestión del conocimiento al modelo de negocio, elaboración de proyectos con base en información explicitada y realización de procesos de seguimiento y monitoreo implementando mejores prácticas.

Por otro lado, las fundaciones empresariales que manifestaron no haber implementado la gestión del conocimiento como proceso efectivo de lo gerencial o administrativo, dicen que esto se debe a que no existen directrices claras por parte de la compañía al respecto, que no hay motivación y que estas actividades se encuentran en proceso de diseño o implementación.

La misma figura sirve para representar el seguimiento o no de las fundaciones empresariales a la gestión del conocimiento, donde igualmente, el 63,6\% manifiesta que no realiza ningún tipo de actividad al respecto. Quienes responden que sí llevan a cabo dicho control, afirman que éste se realiza por medio de auditorías institucionales y de procesos de seguimiento, monitoreo y evaluación empresarial, siendo este último el más mencionado. 

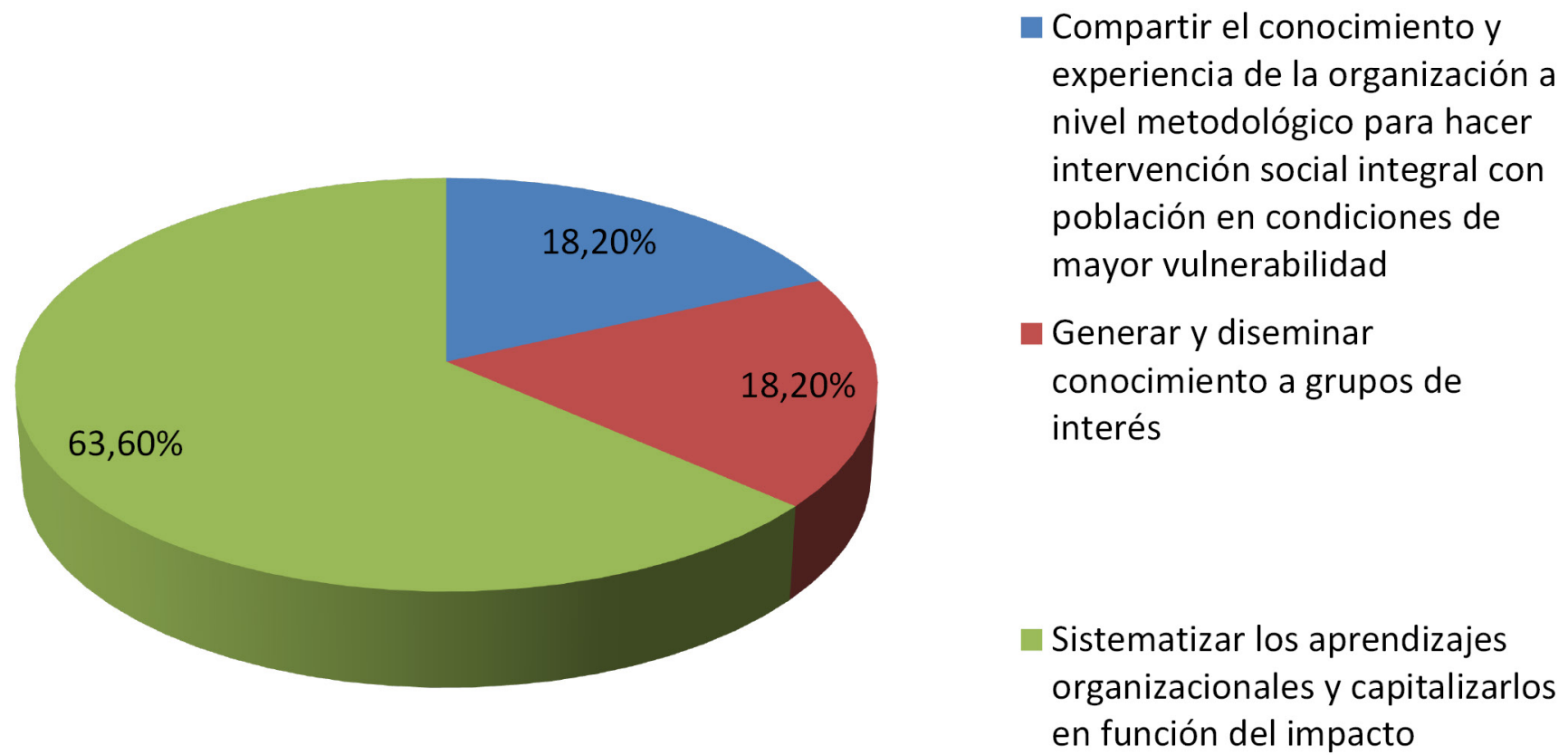

Figura 5. Principales aportes de la gestión del conocimiento al objetivo misional de la fundación.

Fuente: Elaboración propia.

Como se puede deducir de todo lo anterior, es evidente la necesidad de generar y consolidar mecanismos para la gestión del conocimiento en las fundaciones, situación que refuerza el objetivo principal del presente estudio.

De otra parte, cuando se les indaga sobre lo que consideran es el principal aporte de la gestión del conocimiento al objetivo misional de la fundación (Figura 5), el 63,6\% expresa que éste consiste en la posibilidad de sistematizar los aprendizajes organizacionales y capitalizarlos en función del impacto.

Cuando se entra a analizar la relación fundación empresarial-comunidad, se tiene que el $100 \%$ de los encuestados y entrevistados considera que los procesos que involucran a la gestión del conocimiento como elemento mediador, permiten desarrollar mejores intervenciones sociales, justificando sus respuestas en afirmaciones como: "se propicia la capitalización de los aprendizajes y la mejora en las interven- ciones", "se posibilita la estandarización de programas y productos, aprovechando las lecciones aprendidas", "se generan reflexiones permanentes, teniendo en cuenta la visión de los diferentes actores sociales", "se corrigen procedimientos, a partir de las lecciones aprendidas", entre otras.

Por otro lado, al indagar acerca de la relación entre la gestión del conocimiento y la innovación social, reconocen que dicha gestión mejora principalmente los aspectos asociados con los métodos empleados, los procedimientos organizacionales, los productos, los servicios y otros aspectos (relacionamiento con clientes, desarrollo de nuevas maneras de intervención social y desarrollo de experiencias en otros territorios) (Figura 6).

Entre tanto, la Figura 7 expone en resumen los elementos de la gestión del conocimiento detectados en las fundaciones empresariales objeto de estudio, con base en las apreciaciones cualitativas detalladas: 

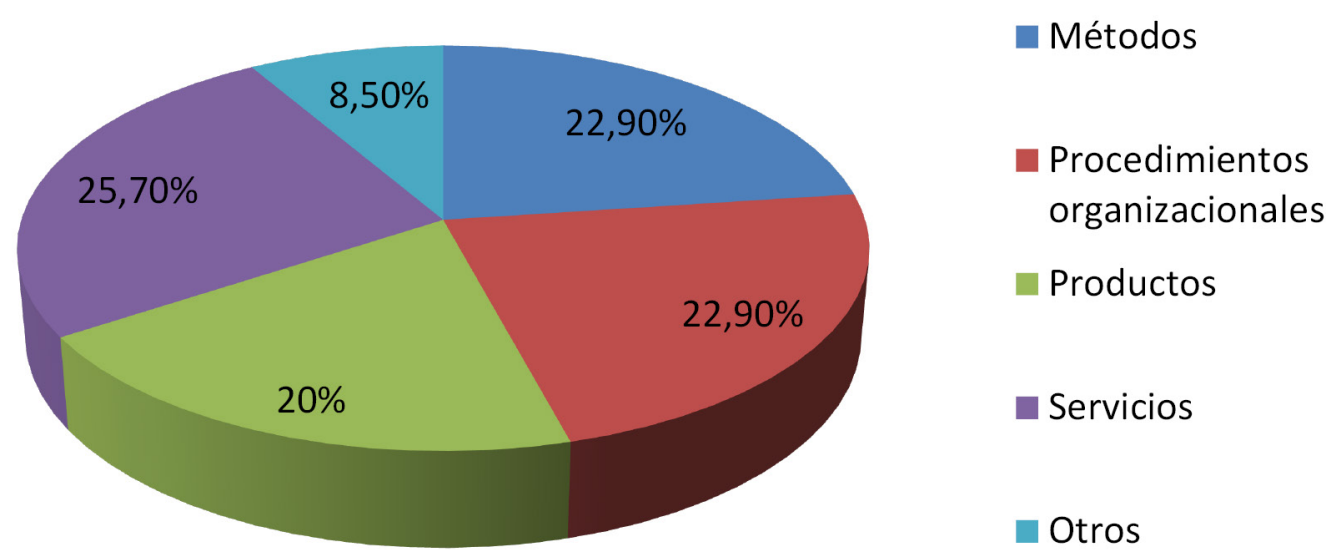

Figura 6. La gestión del conocimiento y su influencia en productos de innovación social. Fuente: Elaboración propia.

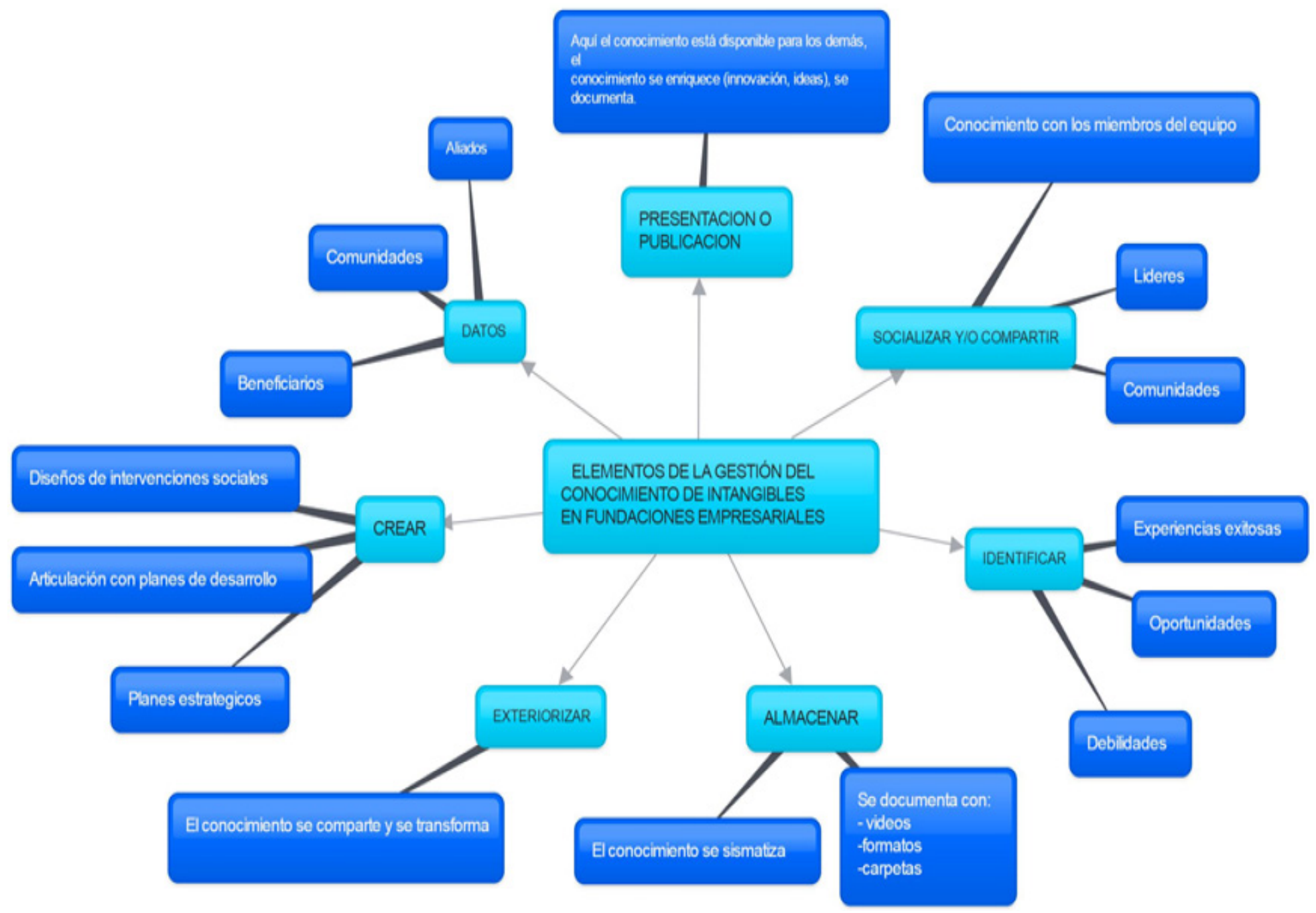

Figura 7. Elementos de la gestión del conocimiento en fundaciones empresariales.

Fuente: Elaboración propia.

Dicha clasificación se origina como resultado de la sistematización de las experiencias descritas, lo que le permite a las fundaciones tener una mirada sistémica de sus intervenciones, de modo que identi- fican, documentan y comunican sus lecciones aprendidas y sus buenas prácticas, sin perder de vista sus elementos constitutivos y los aspectos conexos asociados a su contexto. 


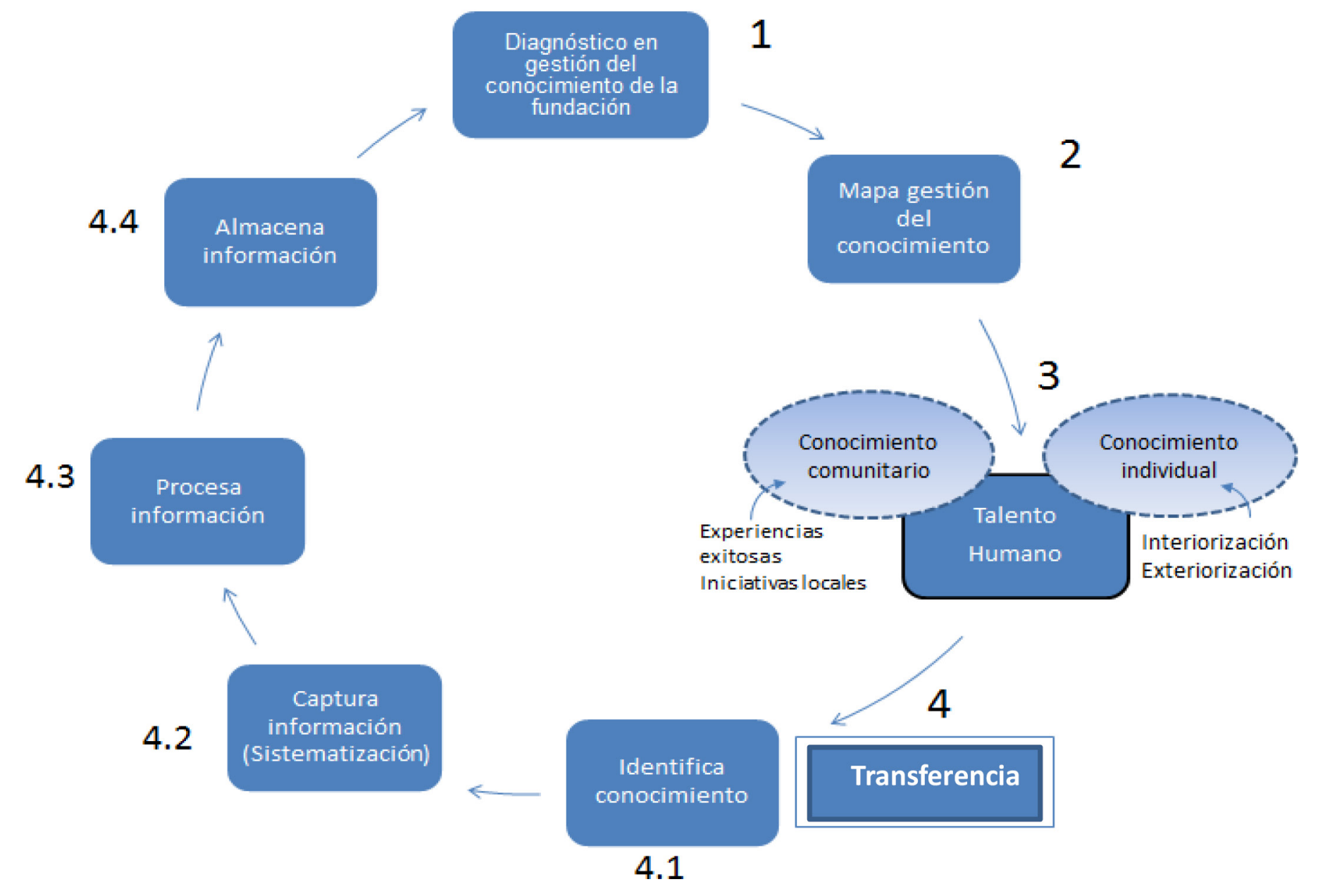

Figura 8. Ruta para la gestión y generación del conocimiento en fundaciones empresariales.

Fuente: Elaboración propia.

Esta información se convierte en el insumo primordial para poder sugerir las posibles fases que deben formar parte de la ruta de gestión y generación de conocimiento para las fundaciones empresariales, lo que constituye el principal aporte de este artículo (Figura 8).

A continuación se describe cada una de estas fases:

1. Diagnóstico de conocimiento. Técnica que permite tener una visión del estado actual de la gestión del conocimiento en las fundaciones, analizando fortalezas, debilidades y oportunidades y amenazas.

2. Mapa de conocimiento. Propicia el acercamiento entre el propósito (estrategia) y el conocimiento natural con que cuentan las fundaciones, identificando sus flujos de conocimiento.
3. Talento humano. Equipo de trabajo que con base en sus competencias y dinámicas puede llevar a cabo el proceso de gestión del conocimiento. Este talento se nutre de dos fuentes: conocimiento comunitario (adquirido a través de encuestas, grupos focales, experiencias exitosas o comunidades de práctica) y conocimiento individual (generado al interior y exterior de la fundación).

4. Transferencia. Proceso a través del cual se comunica la gestión del conocimiento, involucrando: identificación del conocimiento, captura (sistematización), procesamiento y almacenamiento de información (metodologías, formatos, espacios, medios físicos, conversaciones, etc.). 
Adicional a lo anterior, se sugiere para cada una de estas fases, involucrar los elementos descritos en la Tabla 1, como son: etapas, insumos, verificadores de conoci- miento (resultados) y responsables, para tener un mejor ordenamiento, ubicación de las etapas del conocimiento y asignación de responsables para cada proceso.

TABLA 1

Ruta de aprendizaje de gestión del conocimiento en fundaciones empresariales (elementos por fase)

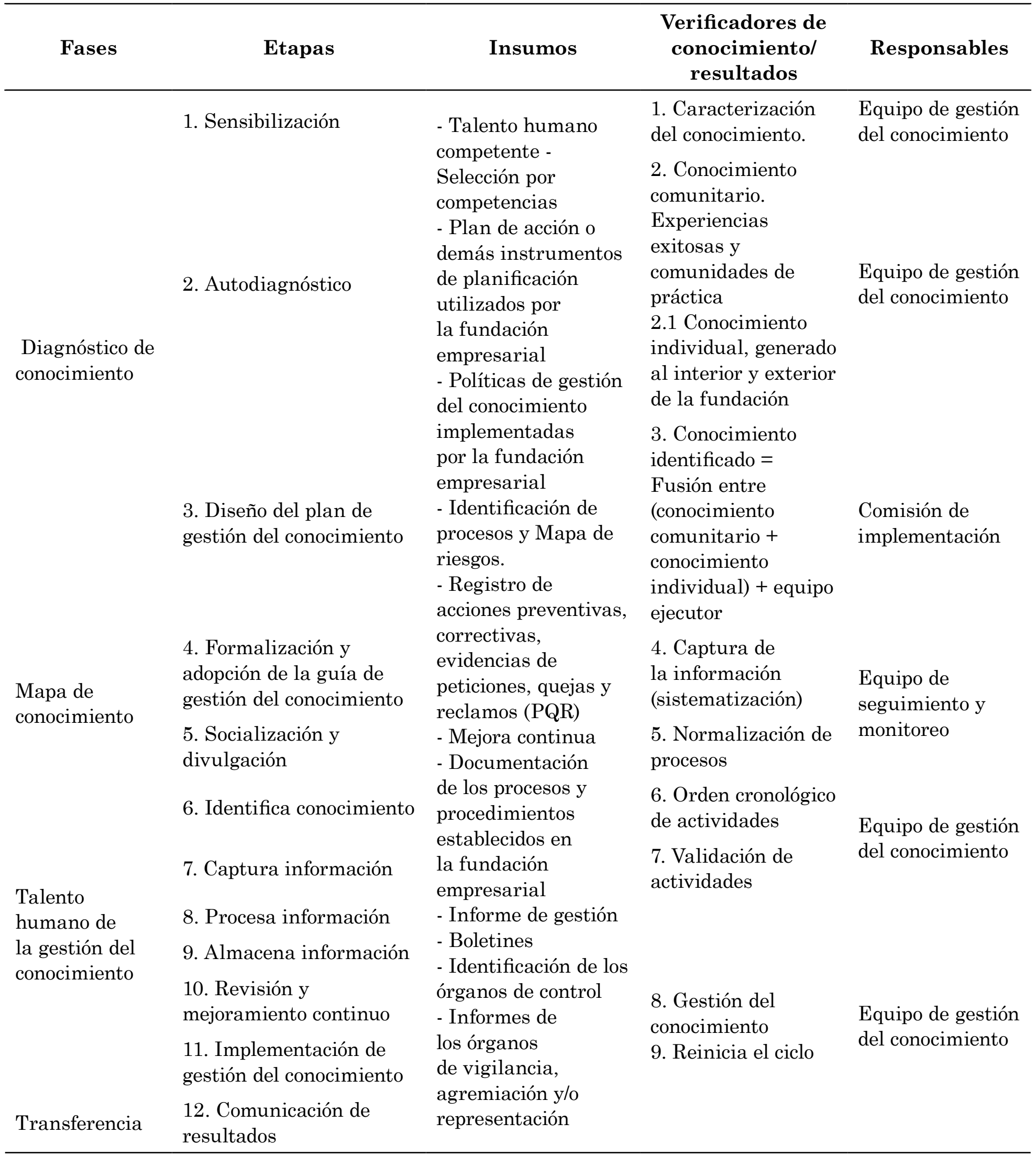

Fuente: Elaboración propia. 
Finalmente, esta ruta tiene dos objetivos: primero, comunicar lo que se entiende por sistematizar una experiencia, y segundo, presentar un derrotero para poder hacerlo. Esperando mejorar el proceso de gestión del conocimiento en las fundaciones empresariales, logrando optimizar sus recursos, convirtiéndose en entidades protagonistas de la organización que las cobija y actores fundamentales para su comunidad de influencia.

\section{DisCUSIÓN (APLICACIÓN)}

A continuación, se somete a consideración la implementación de la ruta de aprendizaje propuesta, tomando como escenario (ejemplo) uno de los procesos asociados a la gestión del conocimiento, analizado en una de las fundaciones objeto de estudio.

En este escenario se determina, a través de la primera fase: diagnóstico de conocimiento, la existencia de debilidades para dicha Fundación, asociadas con la poca preservación del capital intelectual, debido a la escasa articulación entre los procesos organizacionales, incluyendo los externos.

Con base en esto, se establece para el mapa de conocimiento, como segunda fase, el permitir encontrar los aspectos que impiden a esos procesos ser realizados adecuadamente, descubriendo como a pesar de las fortalezas de la Fundación asociadas a sus intenciones para con la comunidad, existen elementos relacionados con la comunicación, principalmente, que impiden a ésta contar con una efectiva memoria organizacional y presenta problemas de pensamiento sistémico, los cuales repercuten en la ausencia de sinergia en algunos de sus procesos, especialmente los externos.

Seguidamente, se define, gracias a la tercera fase (intervención del talento humano), una solución como la concientización de su capital humano, por la importancia de fortalecer sus nexos comunicativos y realizar campañas tendientes a comunicar más efectivamente lo que la Fundación, y hasta la organización, realiza. Esto con la intención de robustecer sus vínculos sociales y cumplirle a la comunidad, según lo establecido por su misión y visión en torno al concepto de servicio, donde la comunicación y las relaciones externas aparecen como elementos importantes.

Finalmente, la Fundación, a través de la transferencia, logra comunicar efectivamente sus acciones, presentándole a la comunidad sus principales actividades asociadas al impacto social, obteniendo, al mismo tiempo, una retroalimentación por parte de dicha comunidad, lo que le permitirá realizar un proceso cíclico de gestión del conocimiento, en el que la transferencia no solo fortalece y fortalecerá su memoria organizacional y sus procesos comunicativos, sino que también permite y permitirá realizar nuevos diagnósticos de conocimiento en pro del desarrollo de la Fundación (su capital intelectual), la compañía y la propia comunidad.

\section{CONCLUSIONES Y RECOMENDACIONES}

Para las fundaciones empresariales estudiadas, los conceptos más importantes asociados a la gestión del conocimiento son: capital humano, flujos de información, medios de interacción y conocimientos tácito y explícito.

Esta claridad permite entender para dichos conglomerados que la gestión del conocimiento se relaciona de manera directa con la apropiación de experiencias, prácticas y lecciones aprendidas; situación que también comprenden las demás organizaciones, pero que a diferencia de éstas, las fundaciones empresariales involucran mucho más la democratización de la información, al poner 
el conocimiento al servicio de todos (tanto de la empresa y la fundación como de la comunidad a la cual impactan o pretenden impactar).

Es así como para la fundación empresarial dichos conceptos y entendimientos le exigen tener que abordar sus procesos de gestión del conocimiento de manera colectiva. Es por esto que las intervenciones sociales empresariales deben estar soportadas en el trabajo colaborativo y en la cooperación, donde el elemento cognitivo se encuentre siempre a merced del capital humano correcto y competente, soportado en la interacción constante entre las personas.

Por lo anterior, se recomienda que las fundaciones empresariales analizadas, $\mathrm{y}$ tal vez en general, consideren la relevancia que tienen los colaboradores externos para llevar a buen término el impacto social de sus procesos, involucrando a la gestión del conocimiento como proceso facilitador $y$ potenciador de sus capacidades.

Para ello, el presente documento propone una ruta de aprendizaje que involucra cuatro fases: diagnóstico inicial del conocimiento (caracterización del estado actual), mapeo del conocimiento (identificación de caminos de flujo), tipificación del talento humano disponible (capital humano responsable de la gestión) y transferencia del conocimiento (identificación, sistematización, procesamiento, almacenamiento y comunicación), para que las fundaciones puedan implementar un proceso viable $\mathrm{y}$ responsable de gestión del conocimiento, que les posibilite detectar a tiempo fallas en sus acciones y mayores posibilidades de éxito en sus procedimientos, con la intención de impactar efectivamente a sus comunidades de contexto y obtener aquel valor diferencial tan necesario en estos tiempos.

\section{REFERENCIAS}

Argyris, C. (1999). On organizational learning. Hoboken: Wiley-Blackwell.

AFE. (2012). Fundaciones empresariales en Colombia: un mundo diverso $y$ dinámico. Bogotá, D.C.: AFE.

Awad, E. M. (2007). Knowledge management. Delhi: Pearson Education India.

Barker, M., Thorne, J. \& Dutnell, M. (1997). Leveraging human capital. Journal of Knowledge Management, 1(1), 63-74. https://doi.org/10.1108/ EUM0000000004581

Barragán, A. (2009). Aproximación a una taxonomía de gestión del conocimiento. Intangible Capital, 5(1), 65-101. https://doi.org/10.3926/ic.2009.v5n1. p65-101

Briceño, M. A. y Bernal, C. A. (2010). Estudios de caso sobre la gestión del conocimiento en cuatro organizaciones colombianas líderes en penetración de mercado. Estudios Gerenciales, 26 (117), 173-193. https://doi.org/10.1016/ S0123-5923(10)70140-6

Canals, A. (2002). Quo Vadis, KM? La complejidad como nuevo paradigma para la gestión del conocimiento. [Working Paper Series WP02-005]. España: IN3. Recuperado de http://www. uoc.edu/in3/dt/20006/index.html

Carcel, F. J. (2014). Planteamiento de un modelo de mantenimiento industrial basado en técnicas de gestión del conocimiento. Valencia: OmniaScienc.

Castells, M. (2009). The rise of the network society: The information age: Economy, society, and culture. Hoboken: WileyBlackwell.

Choo, C. W. (1999). La organización inteligente. El empleo de la información para dar significado, crear conocimiento y tomar decisiones (Rey, D., Trad.). México D.F.: Oxford University Press. 
Chumaceiro, A., Hernández, J., Meléndez, Y. y Acurero, M. (2017). Responsabilidad universitaria y sociedad del conocimiento para la generación de competencias TIC. En, A. Chumaceiro, F. Ramos y J. Hernández (ed.), Procesos formativos para el siglo XXI. Cabimas: Fondo editorial UNERMB.

Davenport, T. H. \& Prusak, L. (2001). Working knowledge: how organizations manage what they know. Boston: Harvard Business School Press.

Drucker, P. (1996). Dirección en instituciones sin fines de lucro. Barcelona: $\mathrm{El}$ Ateneo.

Fundación Carvajal. (2016). Informe de gestión Fundación Carvajal (20142015). [Online]. Recuperado de http:// www.fundacioncarvajal.org.co/wpcontent/uploads/2016/10/informe-degestion.pdf

Fundación Promigas. (2015). Informe de gestión 2015: aprendizajes y transformaciones. [Online]. Recuperado de http://www.fundacionpromigas.org.co/ informegestion2015/

Fundación Saldarriaga Concha. (2017). Gestión del conocimiento. [Online]. Recuperado de http://www.saldarriagaconcha.org/es/gestion-del-conocimiento

Goh, S. C. (2002). Managing effective knowledge transfer: an integrative framework and some practice implications. Journal of Knowledge Management, 6(1), 23-30. http://dx.doi. org/10.1108/13673270210417664

Gorelick, C., Milton, N. \& April, K. (2004). Performance through learning. Knowledge management in practice. New York: Elsevier Butterwork-Heinemann.

Hausmann, R. (noviembre 4, 2013). La economía del conocimiento tácito. El Tiempo. [Online]. Recuperado de http://www.eltiempo.com/archivo/documento/CMS-13159984
Hernández, J. y Chumaceiro, A. (2008). Sociedad del conocimiento y pertinencia social universitaria. CEISEP una propuesta para la UNERMB. Revista Venezolana de Ciencias Sociales, UNERMB, 12(1), 77-92.

Hernández, R., Fernández, C. y Baptista, M. P. (2014). Metodología de la investigación (6 ed.). México D.F.: McGraw-Hill.

Kakabadse, N., Kakadse, A. \& Kouzmin, A. (2003). Reviewing the knowledge management literature: Towards a taxonomy. Journal of Knowledge Management, 7(4), 75-91. https://doi. org/10.1108/13673270310492967

King, W. R. (2009). Knowledge management and organizational learning. Annals of Information Systems, 4(1), 3-13.

Lesser, E. L. \& Storck, J. (2001). Communities of practice and organizational performance. IBM Journal Research and Development, 40(4), 831-841.

Londoño, F. W. y Castillo, F. (2013). Un modelo curricular flexible desde el enfoque sistémico para la formación en Ingeniería de Sistemas en Colombia. En, Innovation in Engineering, Technology and Education for Competitiveness and Prosperity, (LACCEI'2013) 17 LACCEI Latin American and Caribbean Conference for Engineering and Technology. Universidad de Quintana Roo. Cancún, México.

Marín, A. C. y Velasco, M. I. (2001). Historias del aprendizaje: una herramienta para el desarrollo organizacional. Estudios Gerenciales, 17(81), 13-20.

Máynez, A. I. y Cavazos, J. (2011). Conocimiento tácito: su transferencia dentro de la organización, como fuente de ventaja competitiva sostenible. Administración y Organizaciones, 14(26), 9-26.

McAdam, R. \& Reid, R. (2001). SME and large organization perception of knowledge management: Comparisons and contrast. Journal of Knowledge Management, 5(3), 231-241. 
Mori, M. P. (2008). Una propuesta metodológica para la intervención comunitaria. Lima: LIBERABIT.

Nonaka, I. \& Takeuchi, H. (1999). La organización creadora de conocimiento. Cómo las compañías japonesas crean la dinámica de la innovación. México D.F.: Oxford University Press.

Olmos, J., Castro, E. \& D'este, P. (2014). Knowledge transfer activities in social sciences and humanities: Explaining the interactions of research groups with non-academic agents. Research Policy, 43(4), 696-706. http://dx.doi. org/10.1016/j.respol.2013.12.004

Piorun, D. (2018). Introducción a la gestión del conocimiento. Buenos Aires: Universidad de Buenos Aires.

Portillo, A. M. (2015). La construcción del conocimiento en la mente humana: del conocimiento implícito al conocimiento explícito. Revista de Ciencias Sociales, Humanas y Artes, 3(1), 3136.

Quadrado, L. y Simone, C. (2011). O uso da história de vida para compreender processos de aprendizagem gerencial. Revista de Administração Mackenzie, 12(4), 44-74. http://dx.doi.org/10.1590/ S1678-69712011000400003

Quiñones, R. y Senior, A. (2014). Inserción laboral de personas con discapacidad en el sector universitario: Una visión gerencial. Económicas CUC, 35(1), 11-26. Recuperado a partir de https:// revistascientificas.cuc.edu.co/economicascuc/article/view/229

Schoemaker, P. J. H. \& Tetlock, P. E. (2017). Building a more intelligent enterprise. MIT Sloan Management Review, 58(3), 28-38.

Silva, J. et al. (2019). Sistema de alerta temprana basado en la minería de datos para identificar patrones de delincuencia. En, Universidad del Pacífico, Sinbig19 (pp. 259-268). Singapur: Springer.
Soret, I. (2007). Modelo de medición de conocimiento y generación de ventajas competitivas sostenibles en el ámbito de la iniciativa "respuesta eficiente al consumidor", (Efficient Consumer Response) ERC. Madrid: Universidad Rey Juan Carlos.

Sukier, H., Hernandez-Fernandez, L., Portillo-Medina, R., Valle-Ospino, A., Garcia, M. y Garcia-Guiliany, J. (2018). Marketing estratégico: una mirada desde el contexto de la empresa familiar. Revista Espacios, 39(44), 1-9.

Stary, C. (2016). Open organizational learning: Stakeholder knowledge for process development. Knowledge Management \& E-Learning, 8(1), 86-108.

Toffler, A. (1990). El cambio del poder. Conocimientos, bienestar y violencia en el umbral del siglo XXI. Barcelona: Plaza\&Janés.

Valencia, M. (2013). Generación y transferencia de conocimiento. Revista Ingeniería Industrial, 34(2), 13-23.

Vásquez, F. E. y Gabalán, J. (2015). Información y ventaja competitiva. Coexistencia exitosa en las organizaciones de vanguardia. El Profesional de la Información, 24(2), 149-156.

Villar, R., Butcher, J. y Gandini, L. (2014). Fundaciones empresariales en México. Monterrey: Instituto Tecnológico y de Estudios Superiores de Monterrey ITESM.

Villasmil, M. y Crissien, T. (2015). Cambio de paradigma en la gestión universitaria basado en la teoría y praxis de la reingeniería, Económicas CUC, 36(1), 245-274. http://dx.doi.org/10.17981/ econcuc.36.1.2015.29

Wenger, E. (2001). Comunidades de práctica: aprendizaje, significado e identidad. Barcelona: Paidos Iberoamerica.

Wenger, E., McDermott, R. \& Snyder, W. M. (2002). Cultivating communities of practice. Boston: Harvard Business School Press. 


\section{Biodata}

Amin Sinisterra-Núñez es Magister en Administración de Empresas de la Universidad Autónoma de Occidente (Colombia). Docente de la Universidad Uniminuto (Buenaventura, Colombia). Consultor y director de proyectos: metodología del banco de proyectos de inversión nacional del Departamento Nacional de Planeación MGA y marco lógico. Consultor y asesor en gestión territorial municipal y departamental, diseño y estructuración de planes de desarrollo. Profesional misional especializado en desarrollo económico FAO Naciones Unidas (Colombia). https://orcid.org/0000-0001-7569-3188

Lourdes Eugenia Osorio-Bayter es Ph.D y Magister en Economía y Administración de la Universidad de Sevilla (España). Docente de la Universidad de La Salle (Bogotá, Colombia), Miembro del Grupo de Investigación en Ciencias Administrativas-GICAD de la UAO. 20 años de experiencia profesional en la academia, sector gobierno, comunidad y empresa. Directora y jurado en proyectos de doctorado y Máster. Participación en procesos de acreditación en alta calidad, registro calificado e internacionalización en Instituciones de Educación Superior. Par evaluador del Departamento Administrativo de Ciencia, Tecnología e Innovación-Colciencias y Par académico del Consejo Nacional de Acreditación-CNA. Representante nacional al Consejo Directivo del Consejo Profesional de Administración de Empresas del MinCIT. https://orcid.org/0000-0003-0994-4350
Jesús Gabalán-Coello es Ph.D en Medición y Evaluación en Educación de la Universidad de Montreal (Canadá). Magíster en Ingeniería con énfasis en Ingeniería Industrial de la Universidad del Valle (Colombia). Consultor y director de proyectos en temas de aseguramiento de la calidad en el Ministerio de Educación NacionalMEN y el Instituto Colombiano para la Evaluación de la Educación-Icfes. Par evaluador del Departamento Administrativo de Ciencia, Tecnología e Innovación-Colciencias. Par académico del Consejo Nacional de Acreditación-CNA. https://orcid. org/0000-0001-7674-8849

Fredy Eduardo Vásquez-Rizo es estudiante de Doctorado en Gestión de la Información y de la Comunicación en las Organizaciones de la Universidad de Murcia (España). Magíster en Ciencias de la Información y Administración del Conocimiento, Instituto Tecnológico de Estudios Superiores de Monterrey-ITESM (México). Docente de la UAO. Ha trabajado en diversos proyectos y productos sobre educación y gestión de la información y del conocimiento. Par evaluador del Departamento Administrativo de Ciencia, Tecnología e Innovación-Colciencias. Coordinador y miembro de los grupos de investigación en Gestión del Conocimiento y Sociedad de la Información, y Educación, respectivamente, de la UAO. https://orcid.org/0000-00031398-6174 